\title{
Three-dimensional Floquet stability analysis of the wake in cylinder arrays
}

\author{
N. K. - R. KEVLAHAN \\ Department of Mathematics \& Statistics, McMaster University, Hamilton L8S 4K1, Canada
}

(Received 27 June 2007 and in revised form 20 September 2007)

Three-dimensional stability of the periodic wake of tightly packed rotated and inline cylinder arrays is investigated for $60 \leqslant R e \leqslant 270$. Results are compared with existing numerical and experimental studies for an isolated cylinder. Numerical Floquet analysis shows that the two-dimensional wakes of the rotated and inline arrays with spacing $P / D=1.5$ become unstable at $R e_{c}=64 \pm 0.5$ and $R e_{c}=132 \pm 1$ respectively. Two-dimensional vortex shedding flow is unlikely in practice for such flows. The dominant spanwise wavelength is $\lambda / D=0.9 \pm 0.1$ for the rotated array at $R e=100$ and $\lambda / D=3.0 \pm 0.1$ for the inline array at $R e=200$. Three-dimensional simulations show excellent agreement with the Floquet analysis for the rotated case, and reasonable agreement for the inline case. The instability mechanism appears to be similar to Mode A for an isolated cylinder, although the structure of the three-dimensional vorticity is different due to the spatial periodicity of the flow. Unlike the isolated cylinder, both array flows are unstable as $\lambda \rightarrow \infty$ (like a thin shear layer). This is the first investigation of three-dimensional wake instability in cylinder arrays, a problem of significant practical and theoretical interest.

\section{Introduction}

The transition to three-dimensionality of vortex shedding in the wake of an isolated circular cylinder was first explained physically by Williamson (1988). His careful laboratory experiments allowed him to identify two distinct spanwise threedimensional instabilities. Mode $\mathrm{A}$ appears at $R e_{c} \approx 190$ and is characterized by dislocations of the primary spanwise vortices and the formation of pairs of streamwise vortices with a spacing of 3 to 4 cylinder diameters. Mode B appears at $R e_{c} \approx 260$ and is characterized by the formation of streamwise vortices with a much closer spacing of about 1 cylinder diameter. Mode A scales on the size of the primary vortex cores, while Mode B scales on the smaller shear layer between the primary vortices. These experimental observations were confirmed by the numerical linear Floquet stability analysis of Barkley \& Henderson (1996).

The goal of this paper is to analyse the transition to three-dimensional vortex shedding in tightly packed cylinder arrays. The cylinder array is a confined bluff body wake, which is a flow of significant practical interest. Abd-Rabbo \& Weaver (1986) used visualization to show that vortex shedding occurs even under conditions of tight packing (i.e. when the pitch to diameter ratio $P / D<3$ ). Owen $(1965)$ had suggested that tight cylinder spacing suppresses vortex shedding. Abd-Rabbo \& Weaver observed two distinct shedding regimes in a square array, depending on whether the mean flow is aligned with the axis of the array (inline), or at $45^{\circ}$ to the axis the array (rotated). Inline arrays are characterized by approximately symmetric 
vortex shedding (roughly satisfying the symmetry $\omega_{z}(x, y, t)=-\omega_{z}(x,-y, t)$ ), while rotated arrays are characterized by alternate vortex shedding. In the twodimensional vortex shedding regime, both arrays have the exact spatio-stemporal symmetry

$$
\omega_{z}(x, y, t)=-\omega_{z}(x,-y, t+T / 2)
$$

where $x$ is aligned with the direction of the mean flow and $T$ is the periodicity of the flow. This is the spatio-temporal symmetry of the two-dimensional base flows used in the Floquet stability analysis of the three-dimensional instability.

Note that the length of the cylinder array wake is limited by the spacing of the cylinders, whereas for an isolated cylinder the wake length is limited purely by fluid dynamics: Moulinec, Hunt \& Nieuwstadt (2004) argued that the wake disappears in tightly packed cylinder arrays due to turbulent diffusion. Cylinder arrays are also characterized by much higher shedding frequencies due to the close spacing of the cylinders: the Strouhal frequency is about 1 for an array with $P / D=1.5$, compared with about 0.2 for an isolated cylinder. Owen (1965) found empirically that the Strouhal number scales with the mean velocity in the gap between the cylinders.

The main question we address in this paper is how differences in the wake structure of the inline and rotated arrays affect the transition to three-dimensionality in the wake. In particular, we are interested in whether the Mode A and Mode B mechanisms persist, and if the linear Floquet analysis is able to correctly predict the spanwise wavelength of the instability. The distinct wake structures of the inline and rotated arrays suggest that different instability modes may be present at the same Reynolds number for the same array geometry. The only difference is the orientation of the mean flow with respect to the array axis. In this respect the cylinder array may be thought of as breaking the rotational symmetry of the isolated cylinder by imposing a fundamental periodic 'crystal' structure on the flow. We also determine the critical Reynolds number for vortex shedding $\left(R e_{c} \approx 45\right.$ for the isolated cylinder), and the statistics of the lift and drag forces before and after the transition to the threedimensional wake.

We do three different types of numerical simulations in order to address these questions. All simulations are pseudo-spectral with periodic boundary conditions in all directions. We first calculate two-dimensional time-periodic base flows. These are then perturbed in the spanwise direction in order to carry out a numerical Floquet stability analysis, as described in Barkley \& Henderson (1996). The Floquet stability analysis gives the growth rate of the most unstable spanwise mode for each spanwise wavelength and Reynolds number. Finally, we do three-dimensional simulations to check whether the Floquet analysis correctly predicts the spanwise structure of the fully developed three-dimensional flow, visualizations of which allow us to characterize qualitatively the form of instabilities (and compare them to the Mode A and Mode B instabilities of the isolated cylinder).

The results presented here help improve our understanding of how the wake structure of vortex shedding flows affects the stability properties of these flows. In particular, we will see to what extent the isolated cylinder can be taken as a general model for different vortex wake flows. This knowledge could improve the design of heat exchangers by allowing engineers to exploit differences in the dynamical and stability properties of inline and rotated arrays at the same Reynolds number. 


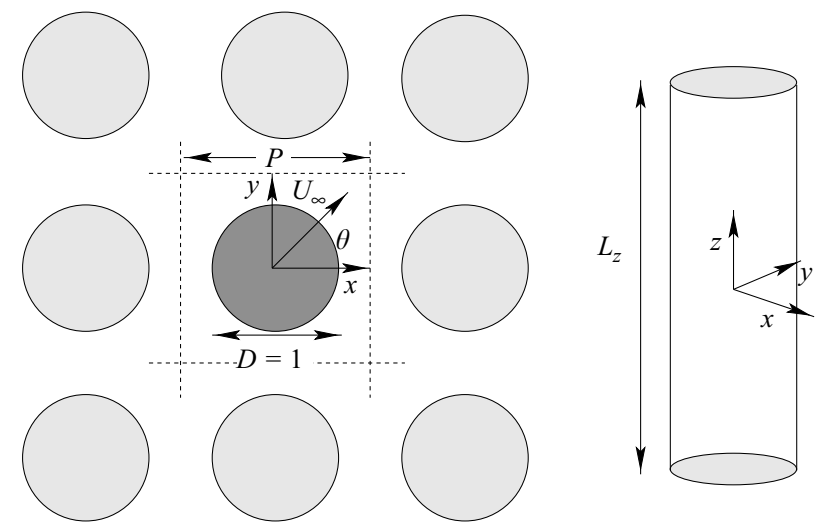

FIGURE 1. Cylinder array configuration. Mean flow angle $\theta=0$ for the inline and $\theta=45^{\circ}$ for rotated arrays. The flow is calculated in the periodic unit cell shown by the dashed lines.

\section{Numerical method}

The two- and three-dimensional flows through inline and rotated square arrays of circular cylinders with a pitch to diameter ratio of $P / D$ are calculated using the following $L_{2}$-penalized equations:

$$
\begin{aligned}
\frac{\partial \boldsymbol{u}}{\partial t}+\boldsymbol{u} \cdot \nabla \boldsymbol{u}+\nabla P & =\frac{1}{R e} \Delta \boldsymbol{u}-\frac{1}{\eta} \chi(\boldsymbol{x}) \boldsymbol{u}, \\
\nabla \cdot \boldsymbol{u} & =0,
\end{aligned}
$$

where the last term on the right-hand side of (2.1) approximates the no-slip boundary conditions on the surface of the cylinder as $\eta \rightarrow 0(\chi(x)=1$ and 0 in the solid and fluid regions of the flow, respectively). This penalized formulation allows a Fourier-based pseudo-spectral method to be used for bluff body flows. Kevlahan \& Ghidaglia (2001) showed that the error in the boundary condition is $O\left(\eta^{1 / 2}\right)$, and the error in the force is $O(\eta)$. We have found that taking $\eta=10^{-4}$ is sufficient for accurate results. The external boundary conditions are periodic in all directions, and we consider a periodic unit cell containing one circular cylinder (as shown in figure 1). We have checked that expanding the periodic unit cell to include two cylinders does not affect the results. The mean velocity over the computational domain, $U_{\infty}=\left\|\boldsymbol{U}_{\infty}\right\|$, is constant (where $\int_{-P / 2}^{P / 2} \int_{-P / 2}^{P / 2} \boldsymbol{u} \mathrm{d} x \mathrm{~d} y=P^{2} \boldsymbol{U}_{\infty}$ ). Note that the integral is taken over the entire computational domain (including the cylinder), which means that $\boldsymbol{U}_{\infty}$ is the mean fluid velocity in the absence of the cylinders (this is the definition used in the cylinder array literature). The Reynolds number $\operatorname{Re}=U_{\infty} D / v$, where $D$ is the cylinder diameter. All quantities are normalized by $D$ and $U_{\infty}$. We consider a tightly packed cylinder array with $P / D=1.5$. The three-dimensional simulations use a spanwise domain $L_{z}=3$ (for rotated arrays) and $L_{z}=6$ (for inline arrays).

Note that the gap velocity $U_{g}$ (i.e. the mean velocity in the narrowest gap between the cylinders) is sometimes used as the reference velocity. $U_{g}$ is significantly higher than $U_{\infty}, U_{g}=U_{\infty} /(1-D / P)=3 U_{\infty}$ if $P / D=1.5$, and this difference should be noted when comparing with other results.

The penalized Navier-Stokes equations (2.1)-(2.2) are solved using a Fouriertransform-based pseudo-spectral method in space (Kevlahan \& Ghidaglia 2001) and a Krylov method in time (Edwards et al. 1994). The pseudo-spectral method is computationally efficient and highly accurate for spatial derivatives, while the Krylov 
method is a stiffly stable explicit method with an adaptive stepsize to maintain the specified error tolerance. The three-dimensional simulations are parallelized as described in Kevlahan \& Wadsley (2005).

The three-dimensional stability of the flow is determined using the Floquet analysis method described in Barkley \& Henderson (1996): a two-dimensional $T$-periodic base flow $\boldsymbol{u}$ is calculated, and then linearly perturbed by spanwise modes

$$
\begin{aligned}
\boldsymbol{u}^{\prime}(x, y, z, t) & =(\hat{u}(x, y, t) \cos \beta z, \hat{v}(x, y, t) \cos \beta z, \hat{w}(x, y, t) \sin \beta z), \\
p^{\prime}(x, y, z, t) & =\hat{p}(x, y, t) \cos \beta z .
\end{aligned}
$$

This defines a linear operator $\boldsymbol{L}$ and an evolution equation for the perturbations

$$
\frac{\partial \boldsymbol{u}^{\prime}}{\partial t}=\mathbf{D N}\left(\boldsymbol{u}, \boldsymbol{u}^{\prime}\right)-\frac{1}{\rho} \nabla p^{\prime}+\frac{1}{R e} \Delta \boldsymbol{u}^{\prime}-\frac{1}{\eta} \chi(\boldsymbol{x}) \boldsymbol{u}^{\prime} \equiv \boldsymbol{L}\left(\boldsymbol{u}^{\prime}\right) .
$$

where $\nabla \cdot \boldsymbol{u}^{\prime}=0, \boldsymbol{u}(x, y, t)$ is the $T$-periodic two-dimensional base flow and

$$
\mathbf{D N}\left(\boldsymbol{u}, \boldsymbol{u}^{\prime}\right) \equiv\left(\boldsymbol{u}^{\prime} \cdot \nabla\right) \boldsymbol{u}+(\boldsymbol{u} \cdot \nabla) \boldsymbol{u}^{\prime}
$$

is the linearized advection term. The solutions of (2.3) are of the form $\tilde{\boldsymbol{u}}(x, y, z, t) \exp \sigma t$, where $\tilde{\boldsymbol{u}}$ are $T$-periodic eigenfunctions (i.e. Floquet modes), and the complex Floquet multipliers $\mu \equiv \exp \sigma T$ are the eigenvalues of $\boldsymbol{L}$. The flow is three-dimensionally unstable to spanwise perturbations of wavenumber $\beta$ if $|\mu|>1$. We are particularly interested in comparing the wavelength and growth rate of the most unstable mode for the isolated cylinder and the cylinder arrays.

We assume that the two-dimensional base flows and flow instabilities are spatially periodic in tightly packed arrays. This assumption is justified by experimental visualization (Abd-Rabbo \& Weaver 1986) and numerical simulations (Moulinec et al. 2004). In this paper we consider only temporal Floquet analysis (based on the time periodicity of the flow). Since the flow is also periodic in space it would also be interesting to consider a full spatial-temporal Floquet analysis (spatial Floquet analysis is described in, e.g., Brevdo \& Bridges (1996)). In fact, the transition to vortex shedding flow could be analysed using spatial Floquet analysis (the results presented here on the critical Reynolds number for the onset of vortex shedding are rough estimates using DNS for a range of Reynolds numbers).

The dominant eigenvalue and eigenfunction are calculated using an Arnoldi iteration scheme with a Krylov subspace dimension of 20 (Barkley \& Henderson 1996), which gives a relative error of $O\left(10^{-3}\right)$ in the calculated value of $\mu$ (based on the exact value $\mu(\beta=0)=1$ ). In each case the base flow is one vortex shedding period saved at 32 times for $t>100$ (when the flow has reached a statistically steady state). The base flow $\boldsymbol{u}$ is obtained at the times required by the Floquet calculation using Fourier interpolation.

The two-dimensional Floquet predictions of the most unstable three-dimensional modes are compared with full three-dimensional numerical simulations. This check is necessary since the linear Floquet mode may be only a transient solution, and does not always describe the fully developed three-dimensional flow.

\section{Results}

\subsection{Characterization of the two-dimensional base flows}

Figure 2 shows a typical wake for the rotated and inline arrays. All two-dimensional simulations were done on a domain of size $1.5^{2}$ with a resolution of $128^{2}$. This 

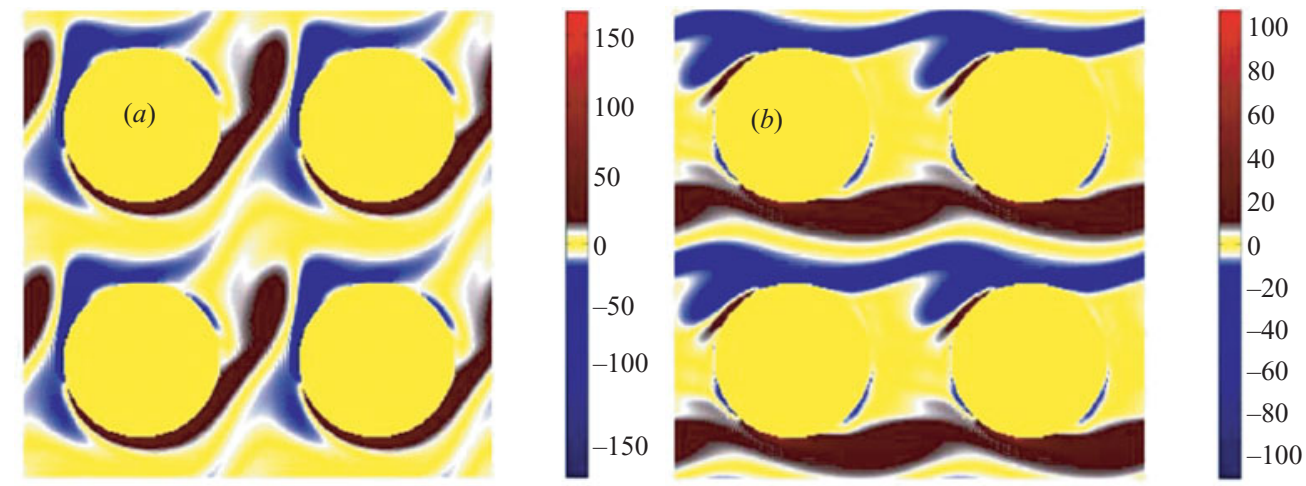

FIGURE 2. Vortex wake structure: instantaneous spanwise vorticity for each configuration. (a) Alternating vortex shedding in the rotated array at $R e=100$. (b) Symmetric vortex shedding in the inline array at $R e=200$.

Case Vortex shedding $R e_{c} \quad 3$-D Floquet $R e_{c} \quad 3$-D Floquet $\lambda_{z} / D \quad\left\langle C_{L}^{2}\right\rangle^{1 / 2} \quad\left\langle C_{D}\right\rangle \quad S t$

$\begin{array}{lcrcccc}\text { Isolated } & 45 & 188.5 \pm 1.0 & 3.96 \pm 0.02 & 0.37 & 1.4 & 0.18 \\ \text { Rotated } & 55 \pm 1 & 64 \pm 0.5 & 0.9 \pm 0.1(R e=100) & 4.9 & 6.8 & 1.24 \\ \text { Inline } & 119 \pm 1 & 132 \pm 1.0 & 3.0 \pm 0.1(R e=200) & 1.4 & 3.0 & 1.08\end{array}$

TABLE 1. Properties of vortex shedding flows (fluid forces and Strouhal numbers are for twodimensional flow at $R e=140)$. $R e_{c}$ and $\lambda_{z} / D$ for the isolated cylinder are from Barkley \& Henderson (1996).

resolution corresponds to a grid size $\Delta x \approx \lambda / 6$ at $R e=200$, where $\lambda=D R e^{-1 / 2}$ is the boundary layer thickness. We have checked that all calculations are fully resolved at this resolution. The periodic unit cell has been copied four times to make the vortex structure clearer. The wake of the rotated array at $R e=100$ shows clearly the alternating vortex shedding pattern observed experimentally by Abd-Rabbo \& Weaver (1986). This wake structure is similar to that of an isolated cylinder, although its downstream extent is limited by the close spacing of the cylinders. Figure $2(b)$ shows the approximately symmetric vortex shedding characteristic of the inline array. The wake pattern is similar to the sinuous mode instability of a triangular jet (Drazin 2002), with the addition of relatively weak symmetric vortex shedding. The triangular jet is unstable for $\lambda / D>0.86$ (taking the gap as the jet width). Recall that both flows are periodic in time, with the exact spatio-temporal symmetry given by (1.1).

Table 1 summarizes some basic dynamical properties of the three vortex shedding flows. The onset of vortex shedding is found to be $R e_{c} \approx 55$ for the rotated array and $R e_{c} \approx 119$ for the inline array. Note that the critical Reynolds number of the rotated array is very close to that of the isolated cylinder $\left(R e_{c} \approx 45\right)$ despite the close cylinder packing (which limits the length of the steady wake). Table 1 also shows that the r.m.s. lift and average drag are much higher for the arrays than for the isolated cylinder, and that the fluid forces are significantly higher in the rotated array than the inline array. As mentioned in $\S 1$ is the Strouhal number for the arrays are also higher than for the isolated cylinder. The values found here are consistent with the empirical fits $S t \approx 1.0$ for the inline array and $S t \approx 1.1$ for the rotated array reported in Weaver, Fitzpatrick \& Elkashlan (1987). 

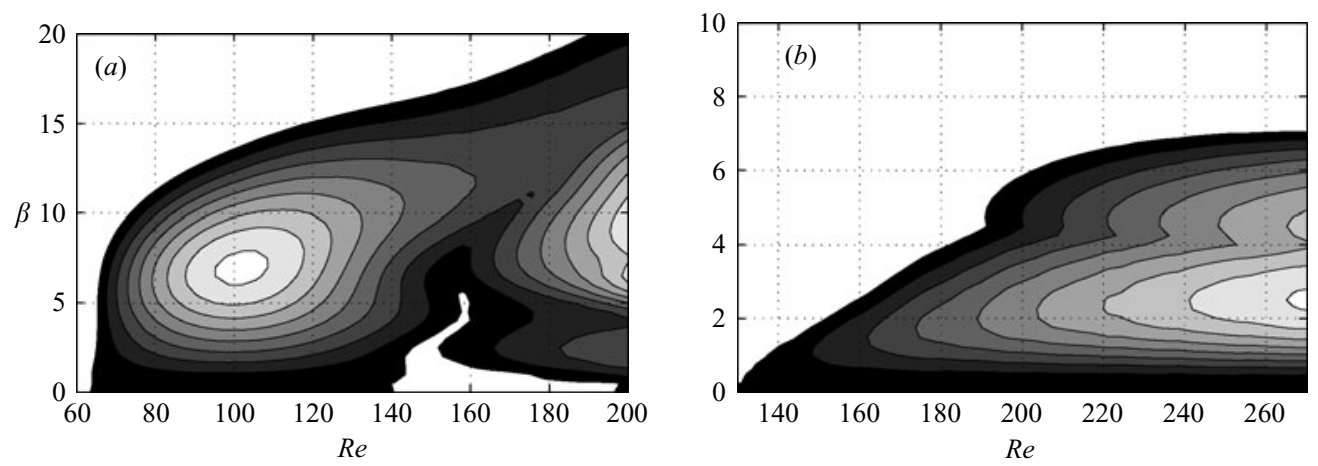

Figure 3. The most unstable Floquet multiplier $|\mu| \geqslant 1$. (a) Rotated, nine equally spaced contours from $|\mu|=1$ to 2 . (b) Inline, nine equally spaced contours from $|\mu|=1$ to 1.4.

\subsection{Three-dimensional Floquet analysis}

Two-dimensional base flows similar to those discussed in $\S 3.1$ were calculated for $60 \leqslant R e \leqslant 200$ for the rotated array and $135 \leqslant R e \leqslant 270$ for the inline array. Beyond these maximum Reynolds numbers the flow is not precisely periodic in time. 32 snapshots of one period were then saved for each Reynolds number and the Floquet stability analysis was done for spanwise wavenumbers $0 \leqslant \beta \leqslant 20$, as described in $\S 2$. We have checked that the $128^{2}$ resolution gives converged results.

The results of the three-dimensional Floquet stability analysis are summarized in table 1. Transition to three-dimensional flow occurs at $R e_{c}=64$ and $R e_{c}=132$ for the rotated and inline arrays respectively. These critical Reynolds numbers are much smaller than previously accepted (e.g. Moulinec et al. (2004) state that the flow in a rotated array is two-dimensional for $R e<200$ ). Both cylinder array flows are significantly more unstable than the flow past an isolated cylinder. In fact, it is likely that two-dimensional vortex shedding flow is not possible in practice (except for very low free-stream turbulence levels). The most unstable three-dimensional wavelength is approximately $1 D$ for the rotated case, and about $3 D$ for the inline case. The spanwise wavelength of the most unstable Floquet mode is close to that of the Mode A instability for the inline array and to that of the Mode B instability for the rotated array. This is surprising since the wake of the inline array is very different from the wake of the isolated cylinder.

Figure 3 shows contour plots of the stability of each array for the whole range of Reynolds numbers and perturbation wavenumbers. Each local maximum along directions of constant Reynolds numbers corresponds to a different Floquet mode. One important difference compared with the isolated cylinder is that the array wakes are unstable for arbitrarily small spanwise wavenumber perturbations (except for the more stable range $140 \leqslant R e \leqslant 190$ in the rotated array). This suggests that confinement makes the wake behave like a thin shear layer or jet, which are similarly unstable for $0<\beta<\beta_{s}$. In contrast, the wake of an isolated cylinder is always stable as $\beta \rightarrow 0$. This difference in behaviour is due to the curvature of the function $|\mu|(\beta)$ at $\beta=0$. For the isolated cylinder, the second derivative of this function is negative, whereas it is positive for both tube array orientations (see figure $5 b$ ). Note also that the rotated array has a much larger unstable region than the inline array.

The dominant (real-valued) Floquet modes of both arrays have the spatio-temporal symmetry of the Mode A instability of an isolated cylinder (Barkley \& Henderson 

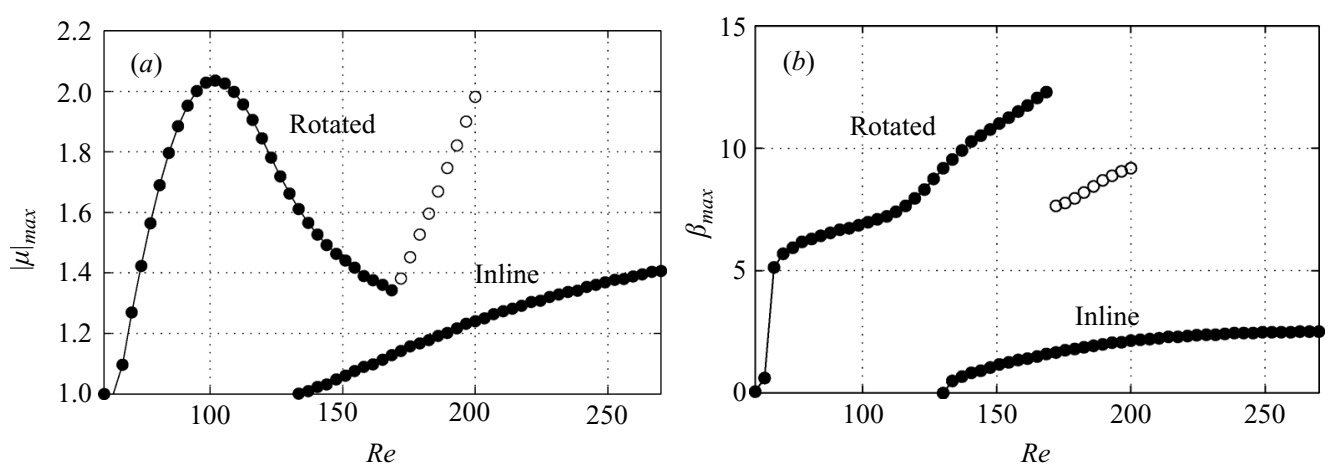

FiguRE 4. (a) Maximum dominant Floquet multiplier as a function of Reynolds number. (b) Wavenumber $\beta$ associated with the maximum dominant Floquet multiplier as a function of Reynolds number. Filled circles indicate real, positive Floquet multipliers $\mu$; open circles indicate complex-conjugate pair multipliers.

1996)

$$
\left.\begin{array}{l}
\omega_{x}(x, y, z, t)=-\omega_{x}(x,-y, z, t+T / 2), \\
\omega_{y}(x, y, z, t)=\omega_{y}(x,-y, z, t+T / 2), \\
\omega_{z}(x, y, z, t)=-\omega_{z}(x,-y, z, t+T / 2),
\end{array}\right\}
$$

where $x$ is aligned with the direction of the mean flow and $T$ is the period of the flow; this is also a symmetry of the two-dimensional base flows (with $\omega_{y}=\omega_{x}=0$ ).

Figure 4(a) shows the maximum growth rate as a function of Reynolds number for both arrays. Note that (as is also clear from figure $3 a$ ), the rotated array has a local instability maximum (corresponding to a positive real-valued Floquet multiplier) near $R e=100, \beta=6.9$. As $R e$ increases further the flow becomes less unstable (with a stable range appearing as $\beta \rightarrow 0$, as mentioned earlier). A second dominant Floquet mode (corresponding to a complex-conjugate pair of Floquet multipliers) appears for $R e>170$. In contrast, the inline array is characterized by a single dominant real-valued Floquet mode that becomes monotonically more unstable with increasing Reynolds number. The discontinuity in the most unstable wavenumber of the rotated array at $R e \approx 170$ shown in figure $4(b)$ is associated with the change in the dominant unstable Floquet mode. The transition to the second dominant Floquet mode is characterized by a jump in the most unstable wavelength from $\lambda_{z}=0.5$ to 0.8 . This mode is clearly seen in figure 3(a) in the form of a second local maximum at $R e=200$. By contrast, figure $4(b)$ shows that the most unstable wavenumber for the inline array plateaus at about $\beta=2.5$ (all dominant $\mu$ are purely real in the inline array).

In figure $5(a)$ we compare the maximum growth rates for the rotated and inline arrays at $R e=200$. Note that the rotated array retains the $\beta=2.1$ (or $\lambda=3 D$ ) realvalued sub-dominant Floquet mode of the inline array in addition to the higher wavenumber $\beta=9(\lambda=0.7 D)$ complex-valued dominant Floquet mode. The stability curves for the rotated array and the isolated cylinder are compared in figure $5(b)$. This shows that although the most unstable spanwise Floquet modes have similar growth rates and wavenumbers, the inline array has a much larger unstable range $(0 \leqslant \beta \leqslant 6.5$ compared with $1 \leqslant \beta \leqslant 2.2$ respectively). As mentioned earlier, a major qualitative difference is that the array flow wakes are generally unstable for arbitrarily small wavenumbers (i.e. arbitrarily large wavelengths). 

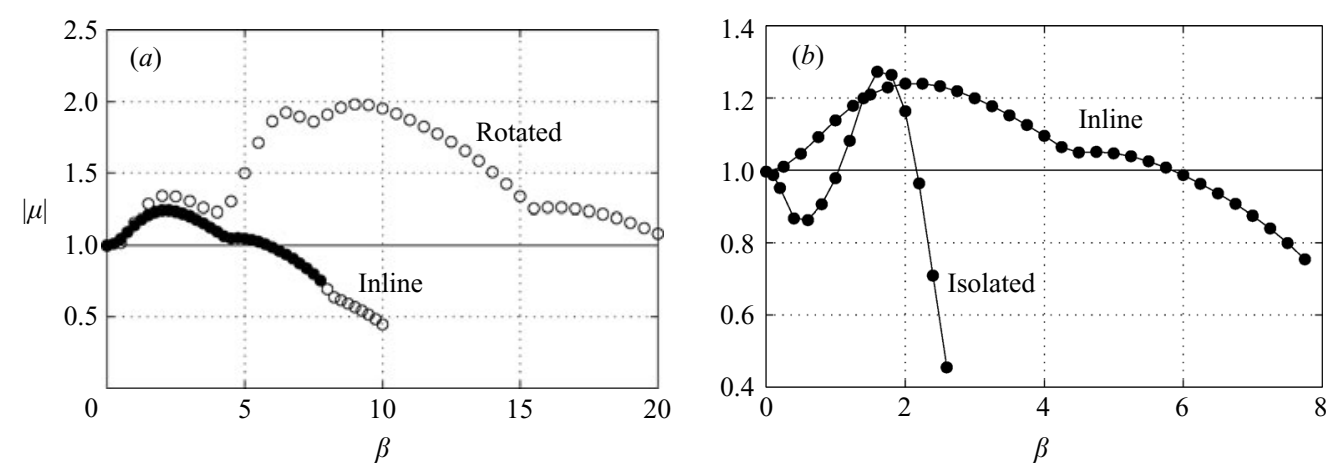

Figure 5. (a) Dominant Floquet multipliers at $R e=200$ for the rotated and inline arrays. (b) Dominant Floquet multipliers at $R e=220$ for the inline array and isolated cylinder (Barkley \& Henderson 1996). Filled circles indicate real, positive Floquet multipliers $\mu$; open circles indicate complex-conjugate pair multipliers.

Taken together, these results demonstrate that rotated and inline arrays have very different three-dimensional stability properties. There is only one dominant threedimensional Floquet mode in inline flow, while the rotated flow exhibits a sudden transition to a new lower wavenumber mode at $R e \approx 170$. This is probably due to differences in the wake structure of the two configurations: the rotated array has an alternate vortex shedding pattern, while the inline array has a jet-like approximately symmetric shedding pattern.

In the following section we use three-dimensional simulations to determine whether the Floquet stability analysis correctly predicts the most unstable spanwise wavelength of fully developed (i.e. nonlinear) three-dimensional flows, and if the wake structures actually correspond to the Modes A and B instabilities of the isolated cylinder.

\subsection{Structure of the three-dimensional instability modes}

In this section we compare the predictions of the linear Floquet analysis with full three-dimensional pseudo-spectral simulations. The simulations were done on domains of $1.5^{2} \times 3($ rotated array at $R e=100)$ and $1.5^{2} \times 6$ (inline array at $\left.R e=200\right)$ with resolutions of $128^{2} \times 64$ and $128^{3}$ respectively. The three-dimensional instability was triggered by initializing the $z$-component of velocity with a random phase $k^{-3}$ spectrum with $1 \%$ of the energy of the mean flow (similar to two-dimensional turbulence and corresponding to the free-stream turbulence present in experiments). We have checked that this resolution gives converged results.

Figure 6 shows the vorticity isosurfaces of all three vorticity components for the rotated and inline arrays. The spanwise wavelength of the three-dimensional instability of the rotated array closely matches the Floquet prediction, $\lambda_{z} / D \approx 1$. The vorticity in the $x, y$ plane is composed of pairs of vortex tubes wrapped around the leading edge of the cylinders. These streamwise vortices alternate in sign in the spanwise direction and in the gap between the cylinders in the $x, y$ plane. This is similar to the structure of the Mode A instability (Williamson 1996) where the streamwise vortices alternate in sign in both the spanwise and streamwise directions. The spanwise vorticity exhibits wavy dislocations similar to those of the Mode A instability for an isolated cylinder. Note, however, that unlike the Mode A instability the vortex tubes are upstream of the cylinder (and wrap around it), rather than extending behind the cylinder in the streamwise direction. 

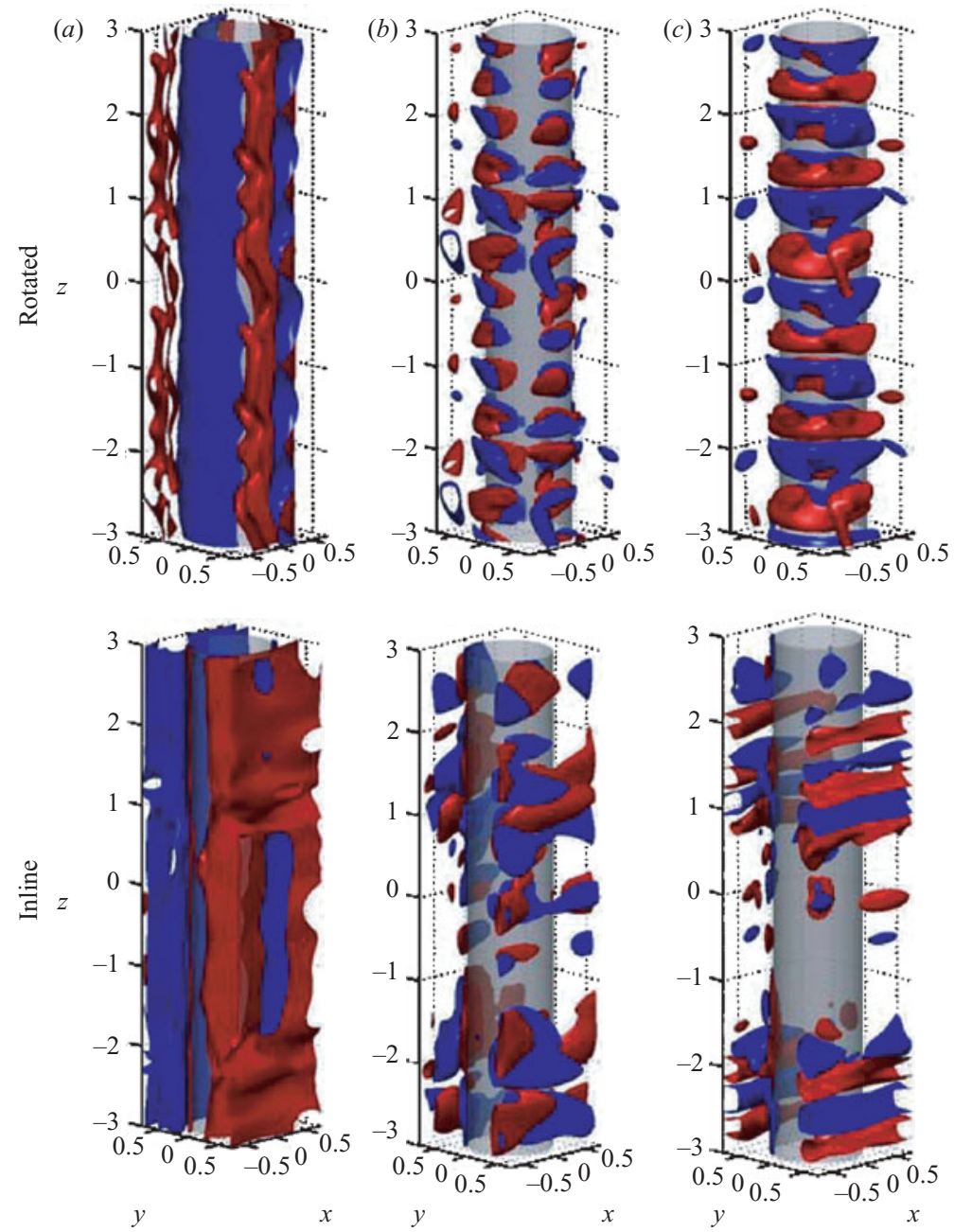

FIGURE 6. Vorticity isosurfaces for the three-dimensional rotated and inline cylinder array flows at $R e=100$ and $R e=200$ respectively: (a) spanwise, $(b)$ streamwise, $(c)$ transverse. The isosurfaces are at $\pm 0.1\|\omega\|_{\infty}$ (spanwise) and $\pm 0.05\|\omega\|_{\infty}$ (streamwise) for the rotated array, and at half these relative values for the inline array. Isosurfaces of positive vorticity are red and isosurfaces of negative vorticity are blue.

The situation is less clear for the inline array. The transverse vorticity isosurface in figure 6 shows two dominant wavelengths: a longer wavelength of $\lambda_{z} / D \approx 3$ and a shorter wavelength of $\lambda_{z} / D \approx 0.7$. The longer one corresponds to the dominant Floquet mode, and the shorter is probably due to nonlinear effects. The transverse vorticity is made up of vortex sheets (rather than vortex tubes). These vortex sheets are arranged in alternating layers, and fill the gap between the cylinders in the transverse direction. This vortex sheet structure is quite distinct from the vortex tube structure of the Mode A or B instabilities of the isolated cylinder. Since the sheets develop relatively late (after $t=50$ ), it is likely that they form by nonlinear vortex stretching. This sheet structure has no equivalent in the wake of an isolated cylinder. 


\section{Conclusions}

This paper presents the first three-dimensional Floquet stability analysis of the wake in cylinder arrays. Besides its direct application to rotated and inline arrays, this study also shows how wake confinement and periodicity affects three-dimensional stability. The main result is that the rotated and inline arrays have very different stability properties at the same Reynolds number, and that two-dimensional vortex shedding flow is probably impossible in practice in tightly packed cylinder arrays. The critical Reynolds number for transition to three-dimensional flow in cylinder arrays previously believed to be about $R e_{c}=200$ (Moulinec et al. 2004) is in fact $R e_{c}=64$.

The Floquet analysis correctly predicts the critical Reynolds number $\left(R e_{c}=64\right)$ and spanwise wavelength (about $1 D$ ) of the three-dimensional instability in the rotated array. The agreement is not as good for inline array, where a smaller wavelength spanwise mode of $0.7 \mathrm{D}$ is evident in addition to the predicted Floquet mode of $3 D$. The dominant real Floquet modes of both arrays have the spatio-temporal symmetry of the Mode A instability of an isolated cylinder. An important result is that both array flows are generally unstable as the spanwise wavelength $\lambda_{z} \rightarrow 0$; this is not the case for the isolated cylinder.

The structure of the streamwise vorticity in the rotated array is similar to that of the Mode A instability of an isolated cylinder. At $R e=200$ the transverse vorticity of the inline array is organized into an unusual vortex sheet structure, quite different from the Mode A and B instabilities. Mode B type instabilities are probably suppressed in arrays since the braid structure is distorted by the tight packing (and the wake includes only one or two vortices).

This study has highlighted differences and similarities in the stability and dynamical properties of confined and open vortex wake flows, and has improved our understanding of the three-dimensional stability properties of confined wakes.

The author thanks Dwight Barkley and Laurette Tuckerman for their useful suggestions. This work was made possible by funding from NSERC and the facilities of SHARCNET.

\section{REFERENCES}

Abd-Rabbo, A. \& Weaver, D. S. 1986 A flow visualization study of flow development in a staggered tube array. J. Sound Vibr. 106, 241-256.

Barkley, D. \& Henderson, R. D. 1996 Three-dimensional floquet stability analysis of the wake of a circular cylinder. J. Fluid Mech. 322, 215-241.

Brevdo, L. \& Bridges, T. J. 1996 Absolute and convective instabilities of spatially periodic flows. Phi. Trans. R. Soc A 354, 1027-1064.

Drazin, P. G. 2002 Introduction to Hydrodynamic Stability, chap. 8. Cambridge University Press.

Edwards, W. S., Tuckerman, L. S., Friesner, R. A. \& Sorensen, D. C. 1994 Krylov methods for the incompressible Navier-Stokes equations. J. Comput. Phys. 110, 82-102.

Kevlahan, N. \& Ghidaglia, J.-M. 2001 Computation of turbulent flow past an array of cylinders using a spectral method with brinkman penalization. Eur. J. Mech. B 20, 333-350.

KeVlahan, N. K.-R. \& Wadsley, J. 2005 Suppression of three-dimensional flow instabilities in tube bundles. J. Fluids Struc. 20, 611-620.

Moulinec, C., Hunt, J. C. R. \& Nieuwstadt, F. T. M. 2004 Disappearing wakes and dispersion in numerically simulated flows through tube bundles. Flow, Turbulence Combust. 73, 95-116.

Owen, P. R. 1965 Buffeting excitation of boiler tube vibration. J. Mech. Engng Sci. 7, 431-439.

Weaver, D. S., Fitzpatrick, J. A. \& Elkashlan, M. 1987 Strouhal numbers for heat-exchanger tube arrays in cross flow. J. Pressure Vessel Tech. 109, 219-223.

Williamson, C. H. K. 1988 Defining a universal and continuous strouhal-Reynolds number relationship for the laminar vortex shedding of a circular cylinder. Phys. Fluids 31, 2742.

Williamson, C. H. K. 1996 Three-dimensional wake transition. J. Fluid Mech. 328, 345-407. 